\title{
COMMENTARY
}

\section{The Need for Nation Wide Electronic Cigarette Smoking Cessation Curricula Across the Healthcare Spectrum}

\author{
Taylor B. Mitchell, Spruha Shah, Lindsay Zink \\ University of North Carolina at Chapel Hill, Eshelman School of Pharmacy, Chapel Hill, North Carolina \\ Corresponding Author: Taylor B. Mitchell, University of North Carolina at Chapel Hill, UNC Eshelman School of Pharmacy, \\ Campus Box 7574, Beard Hall, Chapel Hill, NC 27599-7574. Tel: 704-473-2912. Email: taylor_mitchell@unc.edu
}

Submitted June 3, 2020; accepted March 9, 2021; ePublished March 2021

The use of electronic nicotine delivery systems (ENDS) has become a stepping stone for smokers in their cessation of tobacco use. Students within the pharmacy curriculum and other health care programs have expressed varying responses as to how likely they are to recommend ENDS based on their knowledge of these devices. Due to varying degrees of education given on these products, student pharmacists responded with a greater sense of negativity in the

recommendation of ENDS to current cigarette smokers. This commentary suggests why student pharmacists embody such recommendations and how students within the health care curricula require more adequate ENDS education in order to not recommend these products.

Keywords: vaping, smoking, electronic cigarettes, education, health care students

\section{BACKGROUND}

The use of ENDS has become an increasing social trend among middle school, high school, and college students across America within the past five years. ${ }^{1}$ ENDS now surpasses the use of traditional cigarettes among high school students alone with a $400 \%$ increase in use from 2013 to $2018 .{ }^{1}$ Such products are claimed to have been used by onefourth of America's youth and young adults due to marketing strategies targeting younger generations with various flavors and sexually appealing ads. ${ }^{2}$ In 2014, $26.8 \%$ of young adults perceived ENDS as very harmful and greater than 50\% believed they contained some harm; however, $20 \%$ of young adults believed such nicotine-containing devices had no harm. ${ }^{2}$ Now in an attempt to reduce the number of young patients using ENDS, marketing tactics have switched their advertisements to focus on individuals who currently smoke traditional cigarettes by claiming such devices are safer and provide a better transition to smoking cessation. ${ }^{2}$

Although studies on the full extent of ENDS use are still in development, such nicotine-containing devices may be more harmful than originally thought. ENDS contain a variety of other chemicals with potential harm on a patient's health, including formaldehyde, flavoring chemicals, vitamin E acetate, THC, and other volatile organic compounds. ${ }^{3}$ Such ingredients have been shown to lead to liver, kidney, and neurologic deficits; broncholitis obliterans and other serious lung diseases; and various forms of cancer. ${ }^{3}$ The lack of FDA regulation and testing of ENDS prevents users from knowing all ingredients contained within them, as products do not have to list all harmful substances on the packaging label. ENDS has also led to the outbreak of 2,807 reported hospitalizations and 68 deaths from e-cigarette, or vaping, product use-associated lung injury (EVALI) around the nation as of February 18, 2020. ${ }^{4}$

\section{Consequences}

If proper education and counseling are not utilized by health care workers for patients on the negative consequences of ENDS, this could further increase the rate of EVALI prevalence and hospital admissions due to health complications. This could also lead to unintended increases in cigarette use, illicit drug use, and developmental changes in patients.

Studies show ENDS use is a gateway to smoking traditional cigarettes, marijuana, and other illicit drugs. ${ }^{1,5}$ This is because some nicotine-containing devices also contain THC, the psychoactive mind-altering compound in marijuana that provides a "high" for users due to dopamine release in the brain. ${ }^{4}$ For those who currently smoke, ENDS are considered to have a lower six month remission rate $(22.5 \%)$ compared to nicotine patches $(93 \%)$ for patients who wish to quit smoking. ${ }^{6}$ This is due to ENDS upholding the behavioral pattern similar to traditional cigarettes. ${ }^{6}$

Nicotine acts in a similar manner to THC in stimulating the brain's reward system. In young children and adults, the release of dopamine can lead to neurologic and developmental effects since the prefrontal cortex is the last portion of the brain to develop at 25 years of age. Compared to adults, adolescents are more likely to develop nicotine addictions due 
to having a greater motivation for rewards, less adversity towards risk, and strong influence by peers. ${ }^{7}$ This is a threatening danger to younger generations, as smoking during adolescence increases the risk of developing psychiatric disorders and cognitive impairment later in life. ${ }^{7}$ This potential addictive risk is also seen in children diagnosed with ADHD. From a 2016 national parent survey published by the CDC, approximately 388,000 and 4 million children between ages 2 to 5 years old and 6 to 11 years old were diagnosed with ADHD, respectively. ${ }^{8}$ Due to ADHD adolescents having impulsive and risk-taking behavior characteristics, they are deemed to have a greater risk for addiction and development of regular smoking habits. ${ }^{9,10}$ ADHD patients also demonstrate an increased attention deficit and loss of impulse control, which can be further exacerbated with nicotine products. ${ }^{9}$

\section{Literature Evaluation}

With the increasing use of ENDS, along with the rise of EVALI and other disease complications, Shar, et al. conducted a study to determine the perception of these devices from various health care students. ${ }^{11} \mathrm{~A} 33$-item questionnaire was used and responses were recorded from 1,152 student pharmacists and other health care students from nursing, public health, optometry, and dental hygiene. Results demonstrated statistically significant responses $(\mathrm{p}<.05)$ from student pharmacists who indicated there is more harm with ENDS use compared with other health care students. ${ }^{11}$ Student pharmacists also had more experience in ENDS counseling with patients ( $47 \%$ vs. 38\%, $\mathrm{p}=.005)$, and were more willing to counsel patients on cigarette cessation $(5.01 \pm 1.06$ vs $4.36 \pm 1.36)$ and ENDS cessation $(4.28 \pm 1.22$ vs $3.88 \pm 1.40)$ when responding on a 6 -point Likert scale $(1=$ strongly disagree, $6=$ strongly agree) compared to other health care students. ${ }^{11}$ This may be attributed to their patient care experiences and student pharmacists feeling they received an adequate level of education to counsel on cigarette and ENDS cessation compared to other health care students. ${ }^{11}$ The education student pharmacists received was not described.

Williams distributed an educational smoking cessation curriculum to all pharmacy schools based on a train-thetrainer format due to this lack of education uniformity. ${ }^{12}$ It is used to varying degrees in pharmacy schools across America, but the lack of confidence by professors and a lack of perceived demand by patients prevents it from being fully incorporated into all pharmacy curricula. ${ }^{12}$ Other health care curricula have implemented smoking cessation programs coined the 5A's model (Ask, Advise, Assess, Assist, and Arrange). ${ }^{13}$ This model uses a mnemonic to guide health care professionals in initiating a brief 10 minute intervention session with patients on how to quit smoking cigarettes; however, the curriculum is taught with a wide variety of teaching styles due to professors expressing low self-confidence in knowledge and doubt in the ability to initiate change in patients. ${ }^{13}, 14$ This insecure foundation on the 5A's model has been shown to lead to improper use in health care practice. ${ }^{15,16}$ One study recorded 38 physicians during their tobacco cessation counseling sessions with $32 \%$ of physicians not asking or assessing the patient's current smoking status, thus defeating the purpose of the 5A's mnemonic. ${ }^{15}$ Another survey conducted on Medicaid enrollees indicated $13 \%$ of providers failed to ask about the patient's smoking status, 35\% failed to give advice on quitting, $49 \%$ failed to assess the patient's willingness to quit, $76 \%$ failed to offer any assistance, and $87 \%$ failed to give follow-up appointments. ${ }^{16}$

With proper education, pharmacists could have a greater impact on patient health \& ENDS cessation than many would think. A study conducted in Los Angeles, CA, demonstrated the impact pharmacists have in patient smoking cessation due to their prevalence, convenience, and availability for counseling within a community. ${ }^{17}$ Of the 86 pharmacists responses collected, $42 \%$ received tobacco cessation training in pharmacy school. ${ }^{17}$ Participating pharmacists were recommended to attended a 60-minute, live, web-based training on how to implement brief smoking cessation interventions with patients when providing nicotine replacement therapies (NRTs). ${ }^{17} \mathrm{~A}$ total of 17,013 NRT start kits were distributed over five months while pharmacists provided an intervention counseling session averaging five minutes. ${ }^{17}$ Information on the number of patients who quit smoking were not collected, but $62 \%$ of pharmacists agreed patients were genuinely motivated to stop tobacco use after their intervention, $41 \%$ believed patients would return to their pharmacy for refills on their NRT products, and 94\% agreed they are better equipped to help patients quit smoking as a result of the program, including those who received training during pharmacy school $(p<.05){ }^{17}$

Improper ENDS educational interventions prevents health care professionals from initiating a meaningful impact on patient lives and reaching their full health potential. This lack of initiation is a strike against a health care professional's oath of upholding the priority of a patient's health, as well as impacting the personal health of the patient and their loved ones. This commentary is a call to action in educating future health care workers and implementing a standard educational curricula system that advises against the use of ENDS and traditional cigarette use. Such education can be achieved when standardized academic interventions are upheld and rigorously implemented to health care students through course work and early immersion experiences. ${ }^{18}$ This article describes evidence-based recommendations of potential solutions to be implemented by dynamic health care systems, universities, and professional students.

\section{Solution}


In seeing the positive patient impact proper education has for student pharmacists, the need for an enhanced ENDS and smoking cessation curriculum is necessary and beneficial for all health care students. This allows students and patients to have a better understanding of the risk-benefit profile of ENDS, and their long-term health consequences. Standardized curriculum can include enhancing the use of the 5A's method, adding motivational interviewing skills in counseling sessions, and providing health care students more patient counseling opportunities. This curriculum addition should be taught to students before their first early immersion experience organized by their respective school to obtain more practice. Such education can include a combined formal teaching session with practice opportunities through cocurricular events, health fairs, and student organization events.

In regards to the effectiveness of the 5A's method many physicians and health care professionals admittedly forget what the 5A's stand for, do not know how to correctly implement it, or lack empathy to initiate a continued interest in the patient's need for smoking cessation. ${ }^{15}$ This training can be more effective if better health care curricula implementation strategies were conducted, such as adjusting the mnemonic to be more memorable and dedicating more time to the education of smoking cessation from cigarettes and ENDS.

Motivational interviewing and encouragement are necessary to assist patients in their ENDS and smoking cessation journey. NOVA Southeastern University's (NSU) College of Psychology provides guidance on how to teach students to ask affirmative and open-ended questions, and how to provide information to those who may not want it. ${ }^{19}$ Eliciting "change talk" has also shown to help patients identify their motivations and guide providers on how to teach patients about the health consequences of their actions. ${ }^{19}$ Motivational encouragement includes affirming the patient's feelings, normalizing a patient's fears, and embracing reflective listening. These examples for health care students and professionals acknowledge a patient's current standing towards smoking cessation and encourages them to begin, and continue, their nicotine cessation journey. These motivational and empathetic interventions are critical for patients due to the addiction electronic and traditional cigarettes bring for all users.

Students need to obtain more smoking cessation experiences within their community and educational curricula due to the lack of counseling opportunities expressed by many health care students. ${ }^{11}$ Potential solutions include health care curricula initiating more hours of rotation in their respective fields, providing practice consultation opportunities to students, or encouraging community involvement. At the UNC Eshelman School of Pharmacy, a new curriculum has been implemented to have students obtain 2,400 hours of experience in various pharmacy rotations before graduating. Early student immersions are thought to lead to greater knowledge retention based on the personal patient interactions and experiences encountered..$^{20}$ The school additionally provides opportunities for students to volunteer outside of the classroom through the Student Health Action Coalition (SHAC). This community opportunity allows a variety of health care students to work together to help underserved patients obtain health care service. These interactions include full patient workup assessments and providing clinical recommendations. Objective structured clinical examinations (OSCE) are also performed by students in patient education courses for students utilize their knowledge in a pretend clinical practice setting with a volunteer patient.

\section{CONCLUSION}

The use of ENDS is an increasing trend across the nation among teenagers, young adults, and current cigarette smokers who desire to quit smoking. Health care students must learn how to properly educate patients on the dangers of ENDS use and why they should not be used as alternative to traditional cigarette cessation. Health care students should be fully informed about these products to make more informed risk-benefit analysis decisions. A standardized educational intervention for all health care students is needed to enhance the 5A's method, implement motivational interviewing with empathic listening, and provide more hands-on experience. Future health care providers will then ultimately be fully equipped to create a healthier future and potentially eliminate ENDS usage.

\section{REFERENCES}

1. Know the Risks: E-cigarettes \& Young People | U.S. Surgeon General's Report. https://ecigarettes.surgeongeneral.gov/. Accessed March 2, 2020.

2. Wang TW, Trivers KF, Marynak KL, et al. Harm perceptions of intermittent tobacco product use among U.S. youth, 2016. J Adolesc Health. 2018; 62(6): 750-753.

3. What Do We Know About E-cigarettes? American Cancer Society website https://www.cancer.org/cancer/cancercauses/tobacco-and-cancer/e-cigarettes.html. Updated January 7, 2020. Accessed March 2, 2020.

4. Outbreak of Lung Injury Associated with E-cigarette Use, or Vaping. Centers for Disease Control and Prevention. https://www.cdc.gov/tobacco/basic_information/e-cigarettes/severe-lung-disease.html. Updated February 25, 2020. Accessed March 2, 2020. 
5. Barrington-Trimis JL, Urman R, Berhane K, et al. E-Cigarettes and future cigarette use. Pediatrics. 2016; 138(1): e20160379.

6. Barbeau AM, Burda J, Siegel M. Perceived efficacy of e-cigarettes versus nicotine replacement therapy among successful e-cigarette users: a qualitative approach. Addict Sci Clin Pract. 2013; 8:5.

7. Goriounova NA, Mansvelder HD. Short- and long-term consequences of nicotine exposure during adolescence for prefrontal cortex neuronal network function. Cold Spring Harb Perspect Med. 2012; 2(12): a012120.

8. Data and Statistics about ADHD. Centers for Disease Control and Prevention. https://www.cdc.gov/ncbddd/adhd/data.html. Accessed March 2, 2020.

9. McClernon FJ, Fuemmeler BF, Kollins SH, et al. Interactions between genotype and retrospective ADHD symptoms predict lifetime smoking risk in a sample of young adults. Nicotine Tob Res. 2008; 10(1): 117-27.

10. Gehricke J-G, Hong N, Whalen CK, et al. Effects of transdermal nicotine on symptoms, moods, and cardiovascular activity in the everyday lives of smokers and nonsmokers with attention-deficit/hyperactivity disorder. Psychol Addict Behav. 2009; 23(4): 644-655.

11. Sahr M., Kelsh S., Otteny A., et al. Pharmacy students' perceptions of electronic cigarettes compared to other health care students. Am J of Pharm Educ. 2020; 84(2): 1-6.

12. Williams D. Preparing pharmacy students and pharmacists to provide tobacco cessation counselling. Drug Alcohol Rev. 2009; 28(5): 533-540.

13. Hyndman K., Thomas R., Patterson, S. et al. Effectiveness of tobacco intervention education in health professional students' practice: a systematic review protocol. JBI Database of Systematic Reviews and Implementation Reports. 2016; 14(6): 79-90.

14. Muramoto ML, Lando H. Faculty development in tobacco cessation: training health professionals and promoting tobacco control in developing countries. Drug Alcohol Rev. 2009; 28(5): 498-506.

15. Champassak SL, Catley D, Finocchario-Kessler S, et al. Physician smoking cessation counseling and adherence to a clinical practice guideline. Eur J Pers Cent Healthc. 2014; 2(4): 477-484.

16. Chase EC, McMenamin SB, Halpin HA. Medicaid provider delivery of the 5A's for smoking cessation counseling. Nicotine Tob Res. 2007; 9(11): 1095-101.

17. Hoch MA, Hudmon KS, Lee L, et al. Pharmacists' perceptions of participation in a community pharmacy-based nicotine replacement therapy distribution program. J Community Health. 2012; 37(4): 848-854.

18. King BA, Dube SR, Homa DM. Smoke-free rules and secondhand smoke exposure in homes and vehicles among US adults, 2009-2010. Prev Chronic Dis. 2013; 10: 120218.

19. Sobell LC, Sobell MB. Motivational techniques and skills for health and mental health coaching/counseling: group therapy for substance use disorders: A motivational cognitive-behavioral approach. https://www.nova.edu/gsc/forms/mi-techniques-skills.pdf. Accessed March 2, 2020.

20. Rodgers PT, Cheng V, Bush AA, at al. Characteristics of significant events identified by pharmacy students while on early immersion pharmacy practice experiences. Pharm Pract. 2019; 17(4): 1571. 